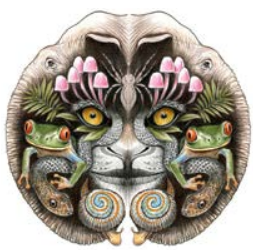

ISSN

Online 0974-7907

Print 0974-7893
Lecanidae is one of the largest and most speciose families of Indian Rotifera (Sharma 1996, 1998). Our recent evaluation of Lecanidae diversity in India (Sharma \& Sharma 2014) highlighted various dubious

OPEN ACCESS lecanid reports in several routine rotifer faunal surveys without any voucher specimens to warrant any validation, while the fuzzy descriptions of new Lecane species from this country and the lack of deposition of their type specimens aggravated taxonomic discrepancies. A compilation of rotifers by Dhanapathi (2000) relying on misidentifications and poor illustrations of the taxon added to such anomalies. This trend has continued unabated in a report on Lecanidae from Andhra Pradesh by Siddiqi \& Karuthapandi (2013). This communication attempts to review the said report with emphasis on misidentifications, nomenclature discrepancies and validity of different species to warrant rectification of anomalous reports and provide a list of valid species from the state.

This validation of Lecane spp. reported by Siddiqi \& Karuthapandi (2013) from Andhra Pradesh is undertaken as a part our ongoing work on 'Monograph of Indian Freshwater Rotifera'. Our comments are based on the said published report vis-à-vis our own examination of Lecanidae collections from different parts of India including those from Andhra Pradesh. The present remarks are supplemented with micro-photographs taken with Leica DM 1000 stereomicroscope fitted with Leica DM 295 image analyzer.

Of the different new records from Andhra Pradesh (Siddiqi \& Karuthapandi 2013, Images 1-6): Lecane aculeata, L. haliclysta, L. furcata, L. pawlowskii, $L$. pyriformis and L. ruttneri; all except L. pyriformis are misidentifications. Lecane bidentata, described by Dhanapathi (1976) from Andhra Pradesh without deposited type, was categorized as species inquirenda by

DOI: http://dx.doi.org/10.11609/JoTT.o3876.5590-2

Date of publication: 26 March 2014 (online \& print)

Manuscript details: Ms \# 03876 | Received 03 December 2013

Citation: B.K. Sharma \& Sumita Sharma (2014). Remarks on 'A report on Lecanidae (Rotifera: Monogononta) from Andhra Pradesh, India': misidentifications and status. Journal of Threatened Taxa 6(3): 5590-5592; http:// dx.doi.org/10.11609/JoTT.03876.5590-2

Copyright: (c) Sharma \& Sharma 2014. Creative Commons Attribution 3.0 Unported License. JoTT allows unrestricted use of this article in any medium, reproduction and distribution by providing adequate credit to the authors and the source of publication.

\section{Remarks on 'A report on Lecanidae (Rotifera: Monogononta) from Andhra Pradesh, India': misidentifications and status}

B.K. Sharma ${ }^{1} \&$ Sumita Sharma ${ }^{2}$

1,2 Freshwater Biology Laboratory, Department of Zoology, North-Eastern Hill University, Permanent Campus, Shillong, Meghalaya 793022, India ${ }_{1}^{1}$ probksharma@gmail.com (corresponding author), 22sumitasharma.nehu@gmail.com

Segers $(1995,2007)$ and Jersabek \& Leitner (2013); it has been placed on the B-list of the "List of available names" (Segers et al. 2012) and as such suggested for removal from zoological nomenclature (http://89.26.108.66/ LAN_CandidatePart-Rotifera-2012-03-22.pdf). Lecane donnerianus Dhanapathi, 1976, described from this state, has been synonymized (Segers 1995) with $L$. ungulata. The unwarranted listing of these taxa as valid species has been continued by Siddiqi \& Karuthapandi (2013). In addition, our remarks on status of various species are summarized in Table 1.

In light of the above remarks, we conclude 22 valid Lecane species known from Andhra Pradesh instead of 33 and 26 species listed by Siddiqi \& Karuthapandi (2013) and Karuthapandi et al. (2013), respectively. These are:

1. Lecane arcula Harring, 1914

2. Lecane bulla (Gosse, 1851)

3. Lecane closterocerca (Schmarda, 1859)

4. Lecane curvicornis (Murray 1913)

5. Lecane eswari Dhanapathi, 1976

6. Lecane furcata (Murray, 1913)

7. Lecane hamata (Stokes, 1896)

8. Lecane hastata (Murray, 1913)

9. Lecane hornemanni (Ehrenberg, 1881)

10. Lecane inopinata Harring \& Myers, 1926

11. Lecane leontina (Turner, 1892)

12. Lecane luna (O. F Muller, 1776)

13. Lecane lunaris (Ehrenberg, 1832)

14. Lecane obtusa (Murray, 1913)

15. Lecane papuana (Murray, 1913)

16. Lecane pyriformis (Daday, 1905)

17. Lecane quadridentata (Ehrenberg, 1832)

18. Lecane stenroosi (Meissner, 1908)

19. Lecane tenuiseta Harring, 1914

20. Lecane tryphema Harring \& Myers, 1926

21. Lecane ungulata (Gosse, 1887)

22. Lecane unguitata (Fadeev, 1925)

Lecanidae of Andhra Pradesh is less species-rich than that documented from several other states of India (Sharma \& Sharma 2014). This misleading generalization is attributed to the fact that Rotifer fauna of this state 
Table 1. Remarks on misidentifications and taxonomic status

\begin{tabular}{|c|c|c|}
\hline & Species & Remarks \\
\hline 1 & Lecane acronycha Harring \& Myers, 1926 & $=$ L. curvicornis (Murray, 1913). Not a synonym of L. acanthinula (Hauer, 1938) (Figs. 1-2) \\
\hline 2 & Lecane aculeata (Jakubski, 1912) & $\begin{array}{l}\text { Misspelled as 'aculeate' in the list and it is a misidentification. Siddiqi \& Karuthapandi (2013, Image 1) } \\
\text { referred to L. arcula Harring, } 1914 \text { (Fig. 3) which distinctly differs from L aculeata (Fig. 4) in size of its } \\
\text { antero-lateral spines. }\end{array}$ \\
\hline 3 & Lecane batillifer (Murray, 1913) & $\begin{array}{l}\text { Questionable record warranting validation; never reported from Andhra Pradesh by Dhanapathi (1976) } \\
\text { as erroneously listed by Siddiqi \& Karuthapandi (2013). This interesting Australasian species is so far } \\
\text { observed (Fig. 5) from India only from its northeast region (Sharma \& Sharma 2014) }\end{array}$ \\
\hline 4 & Lecane bidentata Dhanapathi, 1976 & $\begin{array}{l}\text { species inquirenda (Segers 1995, 2007; Jersabek \& Leitner 2013); definitely not even distantly related to } \\
\text { and not a synonym of L. batillifer (Murray, 1913) }\end{array}$ \\
\hline 5 & Lecane vasishti Sharma, 1980 & $\begin{array}{l}\text { Misspelled as 'vasisthi' - a distinct species (Sharma \& Sharma 2014) so far known only from its 'type } \\
\text { locality' in West Bengal (Figs. 6-7); not a synonym L. crepida Harring, } 1914 \text { (Fig. 8) and it differs distinctly } \\
\text { from the same. }\end{array}$ \\
\hline 6 & Lecane donnerianus Dhanapathi, 1976 & $=$ L. ungulata (Gosse, 1887); not a synonym of L. donneri Chengalath \& Mulamoottil, 1974 \\
\hline 7 & Lecane furcata (Murray, 1913) & $\begin{array}{l}\text { A misidentification; Siddiqi \& Karuthapandi (2013, Image 5) apparently showed L. lunaris (Fig. 9) and it } \\
\text { differed distinctly from L. furcata (Fig. 10) }\end{array}$ \\
\hline 8 & Lecane haliclysta Harring \& Myers, 1926 & $\begin{array}{l}\text { A misidentification; Siddiqi \& Karuthapandi (2013, Image 5) apparently referred to L. tenuiseta Harring, } \\
1914 \text { (Fig. 11) }\end{array}$ \\
\hline 9 & Lecane lauterborni (Hauer, 1924) & $\begin{array}{l}\text { Reported by Dhanapathi (1976) from Andhra Pradesh. It is a case of misidentification (refer: Segers \& } \\
\text { Savatenalinton 2010; Sharma \& Sharma 2014) }\end{array}$ \\
\hline 10 & Lecane pawlowskii Wulfert, 1966 & $\begin{array}{l}\text { A misidentification and misspelled as 'Walfert'; Siddiqi \& Karuthapandi (2013, Image 3) appeared to refer } \\
\text { to a partly contracted L. closterocerca (Fig. 12). Lecane pawlowskii - an Indian endemic (Figs. 13-15) is } \\
\text { known till date from Gujarat (type-locality) and West Bengal (Sharma \& Sharma 2014). }\end{array}$ \\
\hline 11 & Lecane ohioensis (Herrick, 1885) & A distinct species and not a synonym of L. obtusa (Murray, 1913) \\
\hline 12 & Lecane ploenensis Voigt, 1902 & $\begin{array}{l}\text { Misspelled as 'ploensis'); not a syn. of L. pideis (Harring \& Myers, 1926) (apparently misspelled) which itself } \\
\text { not any valid species }\end{array}$ \\
\hline 13 & Lecane ruttneri (Hauer, 1938) & A misidentification as Siddiqi \& Karuthapandi (2013, Image 4) apparently showed L. hornemanni (Fig. 16) \\
\hline 14 & Lecane tethis (Harring \& Myers, 1926) & $=$ L. furcata (Murray, 1913); not a synonym of L. tenuiseta Harring, 1914 \\
\hline 15 & Lecane stichoclysta Segers, 1993 & Never reported from Andhra Pradesh by Dhanapathi (1976) and not known till date from India \\
\hline 16 & Lecane styrax (Harring \& Myers, 1926) & A distinct species, not a synonym of Lecane stichoclysta Segers, 1993 \\
\hline
\end{tabular}

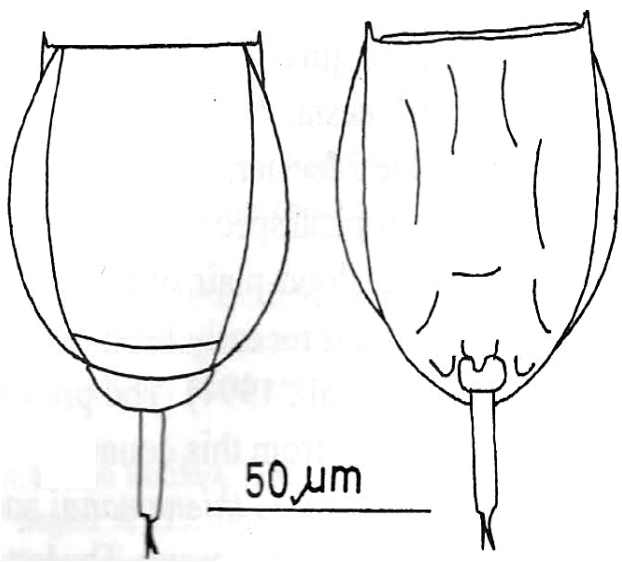

Figures 1-2. Lecane acanthinula (Hauer)

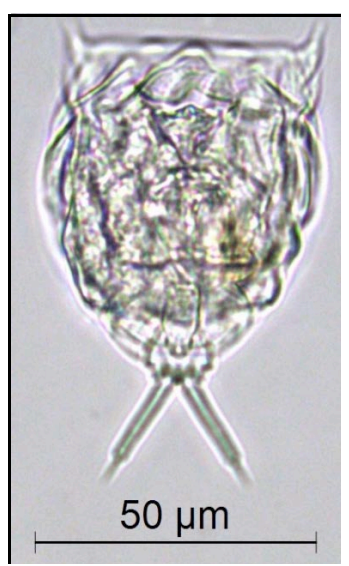

Figure 3. Lecane arcula Harring

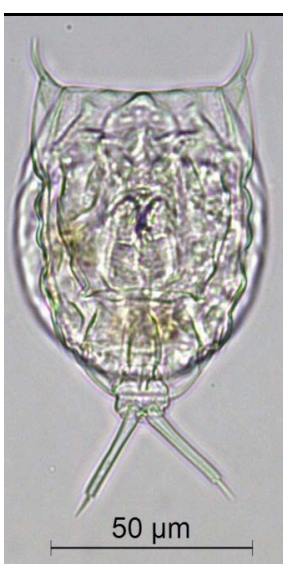

Figure 4. Lecane aculeata (Jakubski)

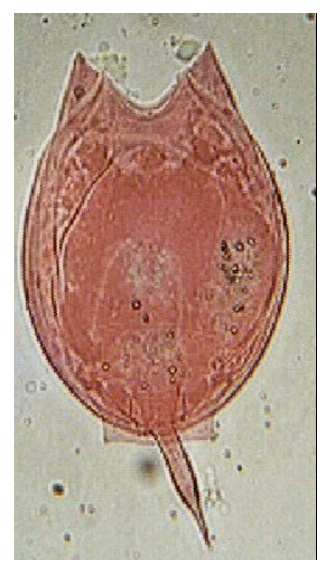

Figure 5. Lecane batillifer Hauer is yet partly explored. Of the documented species, $L$. eswari merits biogeographic interest as this erstwhile Indian endemic is a Paleotropical species (Sharma \& Sharma 2009, 2014; Segers \& Savatenalinton 2010)

\section{References}

Dhanapathi, M.V.S.S.S. (1976). Rotifers from Andhra Pradesh, India - III. Family Lecanidae including two new species. Hydrobiologia 48(1): 9-16.

Dhanapathi, M.V.S.S.S. (2000). Taxonomic notes on the Rotifers from India (from 1889-2000). Indian Association of Aquatic Biologists (IAAB), publication No. 10. Hyderabad, 1-180pp.

Jersabek, C.D. \& M.F. Leitner (2013). The Rotifer World Catalog. World 


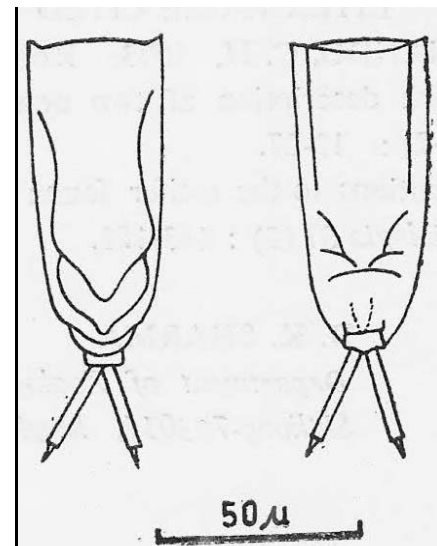

Figures 6-7. Lecane vasishti Sharma

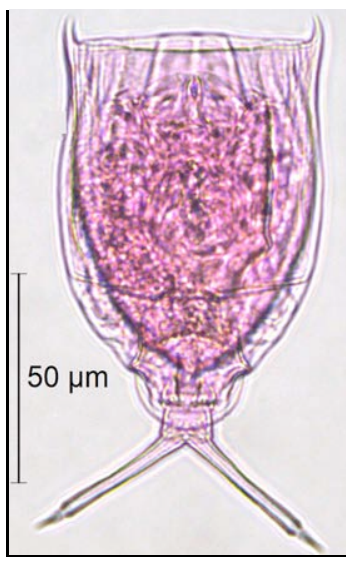

Figure 8. Lecane crepida Harring

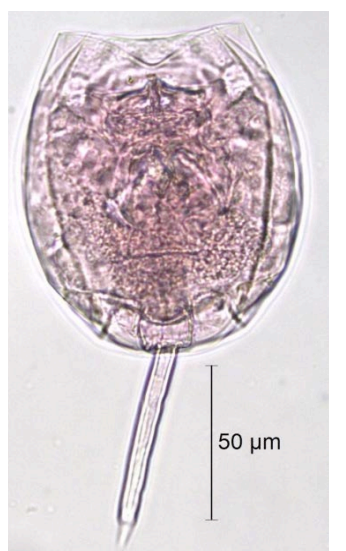

Figure 9. Lecane lunaris (Ehrenberg)

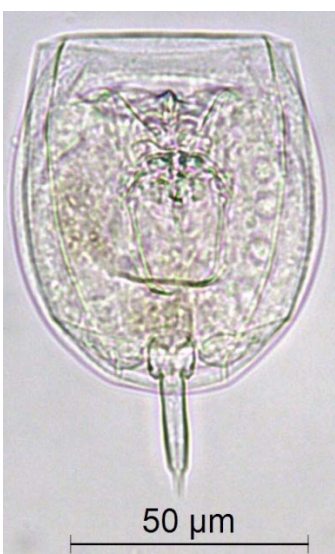

Figure 10. Lecane furcata (Murray)

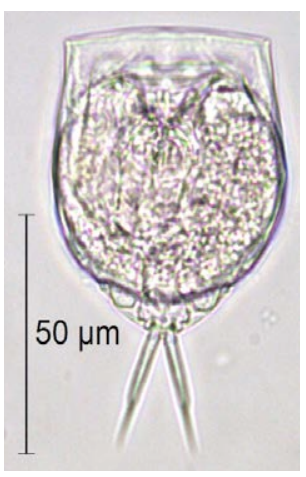

Figure 11. Lecane tenuiseta Harring, 1914

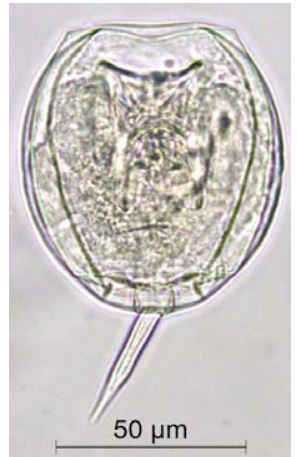

Figure 12. Lecane closterocerca (Schmarda)
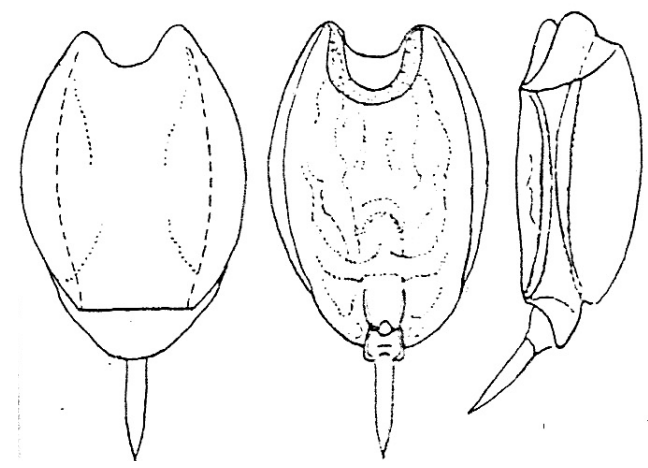

Figures 13-15. Lecane pawlowski Wulfert (after Wulfert, 1966)

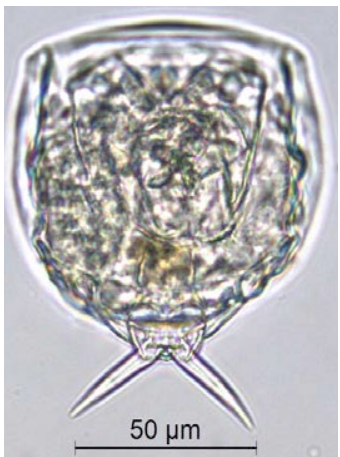

Figure 16. Lecane hornemanni (Ehrenberg)
Wide Web electronic publication <http://www.rotifera hausdernatur.at/> accessed on 03.12.2013.

Karuthapandi, M., D.V. Rao \& X. Innocent (2013). Freshwater rotifers of Andhra Pradesh- checklist. International Journal for Life Sciences and Educational Research 1(1): 1-13

Segers, H. (1995). Rotifera 2: The Lecanidae (Monogononta). In: Dumont H.J. \& T. Nogrady (eds.). Guides to Identification of Microinvertebrates of the Continental Waters of the World. SPB Academic Publishing bv. Amsterdam, Netherlands, 226pp.

Segers, H. (2007). Annotated checklist of the rotifers (Phylum Rotifera) with notes on nomenclature, taxonomy and distribution. Zootaxa 1564: 1-104.

Segers, H. \& S. Savatenalinton (2010). A critical re-revaluation of the Lecanidae (Rotifera: Monogononta) of Thailand, with description of a new species. International Review of Hydrobiology 95: 343-351.

Segers, H., W.H. de Smet, C. Fischer, D. Fontaneto, E. Michaloudi, R. L. Wallace \& C.D. Jersabek (2012). Towards a list of available names in zoology, partim Phylum Rotifera. Zootaxa 3179: 61-68.
Sharma, B.K. (1996). Biodiversity of freshwater rotifera India - a status report. Proceedings of the Zoological Society 49(2): 73-85.

Sharma, B.K. (1998). Faunal diversity in India: Rotifera, pp. 57-70. In: Alfred, J.R.B., A.K. Das \& A.K. Sanyal (eds.). Faunal Diversity of India ENVIS Centre, Zoological Survey of India, Calcutta.

Sharma, B.K. \& S. Sharma (2009). Biodiversity and distribution of freshwater rotifers (Rotifera, Eurotatoria) of Tamil Nadu. Records of the Zoological Survey of India 109(3): 41-60.

Sharma, B.K. \& S. Sharma (2014). Northeast India: an important region with a rich biodiversity of Rotifera. International Review of Hydrobiology 99: 20-37; http://dx.doi.org/10.1002/iroh.201301701

Siddiqi, S.Z. \& M. Karuthapandi (2013). A report on Lecanidae (Rotifera: Monogononta) from Andhra Pradesh, India, including six new distribution records with notes on their contemporary taxonomic nomenclature. Journal of Threatened Taxa 5(11): 45564561; http://dx.doi.org/10.11609/JoTT.03095.4556-61 